\title{
Development of an Aircraft Auto-landing Guidance System using Time Delay Control, and Simulation with Crosswind and Aileron Fault*
}

\author{
Hyoung Sik Chor, ${ }^{1)}$ Sangjong LeE, ${ }^{2)}$ Jangho LeE, ${ }^{1)}$ Hyeok Ryu, ${ }^{1)}$ Hyunchul Shim,${ }^{3)}$ and Cheolkeun $\mathrm{HA}^{4)}$ \\ ${ }^{1)}$ Korea Aerospace Research Institute, Daejeon 305-806, Republic of Korea \\ ${ }^{2)}$ Department of Aircraft Mechanical Engineering, Inha Technical College, Incheon 402-752, Republic of Korea \\ ${ }^{3)}$ Department of Aerospace Engineering, Korea Advanced Institute of Science and Technology, Daejeon 305-701, Republic of Korea \\ ${ }^{4)}$ Department of Aerospace Engineering, University of Ulsan, Ulsan 680-749, Republic of Korea
}

A design procedure for aircraft auto-landing guidance using time delay control (TDC) is proposed, and the designed guidance system is validated via simulations of general landing procedures, including glide slope, flare and touchdown. The simulations adopt a nonlinear dynamics model that includes the landing gear. Additionally, by employing TDC, a stability and control augmentation system, a longitudinal auto-landing guidance law, and a lateral guidance law considering crosswind are proposed. The designed auto-landing guidance laws are evaluated through simulations with aileron fault and crosswind disturbance. The proposed TDC-based auto-landing guidance law shows good performance and is robust when aileron fault and crosswind are included.

Key Words: Aircraft, Auto-landing, Time Delay Control, Aileron Fault, Crosswind

\section{Nomenclature}

$\alpha, \beta$ : angle of attack and sideslip

$\phi, \theta:$ roll and pitch angle

$u, v: x, y$ velocity components in body axis

$p, q$ : roll and pitch rate

$h$ : altitude

$d$ : lateral offset from reference line

$V$ : aircraft velocity in earth frame

$V_{N}, V_{E}$ : north and east ground velocity

$\bar{l}, \bar{M}$ : rolling and pitching moment

$\psi$ : heading angle

$\Psi^{\prime}$ : course angle, $\tan ^{-1}\left(V_{E} / V_{N}\right)$

$M_{\delta_{e}}$ : elevator control moment

$l_{\delta_{a}}:$ aileron control moment

$I_{x, v}$ : moment of inertia in roll, pitch axis

$\Lambda$ : line of sight angle

$\delta_{a}, \delta_{e}$ : aileron and elevator deflection angle

$\gamma$ : flight path angle

$g$ : gravity acceleration parameter

$L$ : small time step

$t$ : time

$\boldsymbol{e}$ : error vector

$\boldsymbol{u}$ : control input vector

$\boldsymbol{x}$ : states vector

$r$ : reference input

Subscripts
0 : initial
$d$ : desired
$r$ : reference command

(C) 2015 The Japan Society for Aeronautical and Space Sciences *Received 16 April 2012; final revision received 12 March 2013; accepted for publication 23 October 2013.

\section{Introduction}

Time delay control (TDC) is a nonlinear model reference control (MRC) that has recently been applied to various nonlinear plants and models with uncertainty in many engineering fields, including mechanics and robotics, and has shown satisfactory performance. ${ }^{1-5)}$ TDC does not require an accurate model, and is known to be robust against disturbances and for modeling uncertainties. ${ }^{3-5}$ )

This technique takes values from the previous step and uses them for feedback. Similar nonlinear control techniques that utilize time delayed observation for feedback have been used $^{6)}$ prior to the late 1980s when Youcef-Toumi and Ito, and Hsia compiled the theories and established this technique.

Youcef-Toumi and Ito first named this technique TDC, ${ }^{7,8)}$ while Hsia proposed a similar methodology at around the same time ${ }^{9,10)}$ without using the same nomenclature. Although their works were slightly different in terms of mathematical formulation, both approaches were identical in the concept that they use time delay for estimation. The similarities and differences between their approaches are clearly summarized in Chang and Lee. ${ }^{11)}$ Later, Youcef-Toumi and $\mathrm{Wu}^{12)}$ applied stability analysis and input/output feedback linearization (IOFL) control to the TDC using a similar formulation as Hsia and Gao. ${ }^{10)}$ The common aspect of the TDCs from Youcef-Toumi and Ito, and Hsia, and the IOFL TDC from Youcef-Toumi and Wu is that they establish plants in the phase variable (canonical) form. The strength of this approach is that uncertainties and disturbances can be eliminated using very simple formulations.

The present paper deals with longitudinal and lateral TDCbased auto-landing guidance laws for an unmanned aerial vehicle (UAV). The authors have previously applied TDC 
for longitudinal auto-landing guidance, and have demonstrated satisfactory robustness of the system against uncertainties in the model and disturbances. ${ }^{13)}$ Further, the feasibility of employing TDC in an actual system was shown through flight tests using TDC SCAS. ${ }^{14)}$ The present paper extends the series of earlier efforts to lateral control loop using the line of sight (LOS) guidance law, and concludes with an auto-landing guidance system. The performance of the TDC-based auto-landing guidance law is evaluated using simulations including aileron fault and steady crosswind.

\section{TDC}

In this section, the concept of TDC is introduced with a formulation that combines the two previous approaches. ${ }^{8,10)}$ The relationship between two approaches are well summarized in Chang and Lee. ${ }^{11)}$

\subsection{Introduction to TDC}

Consider a time varying nonlinear dynamic equation with a disturbance, $\boldsymbol{d}(t)$, as ${ }^{11)}$ :

$$
\dot{\boldsymbol{x}}=\boldsymbol{f}(\boldsymbol{x}, t)+\boldsymbol{B}(\boldsymbol{x}, t)+\boldsymbol{d}(t)
$$

If the exact value of every term in Eq. (1) is known and $\boldsymbol{B}(\boldsymbol{x}, t)$ is perfectly inverse transformable, the input, $\boldsymbol{u}$, can be found by inverting the model as:

$$
\boldsymbol{u}=\boldsymbol{B}^{-1}(\boldsymbol{x}, t)[\dot{\boldsymbol{x}}-\boldsymbol{f}(\boldsymbol{x}, t)-\boldsymbol{d}(t)]
$$

However, in reality, direct inverse transformation is not reliable because the exact value of $\boldsymbol{f}(\boldsymbol{x}, t)$ and $\boldsymbol{B}(\boldsymbol{x}, t)$ cannot be determined. Therefore, a pseudo control $(\boldsymbol{v}(t))$ is employed and the model is sequentially inverted.

First, Eq. (1) is rearranged as:

$$
\dot{\boldsymbol{x}}=\boldsymbol{h}(\boldsymbol{x}, t)+\hat{\boldsymbol{B}} \boldsymbol{u}
$$

where, $\hat{\boldsymbol{B}}$, a constant or a matrix with constant elements, is an estimate of the control effectiveness $\boldsymbol{B}(\boldsymbol{x}, t)$ within a predictable bound. A new nonlinear equation $(\boldsymbol{h}(\boldsymbol{x}, t))$, which includes all the nonlinearities, disturbances and uncertainties, is expressed as:

$$
\boldsymbol{h}(\boldsymbol{x}, t)=\boldsymbol{f}(\boldsymbol{x}, t)+[\boldsymbol{B}(\boldsymbol{x}, t)-\hat{\boldsymbol{B}}] \boldsymbol{u}+\boldsymbol{d}(t)
$$

With the pseudo control, $v$, defined in Eq. (5), the input computed by inverting the model yields Eq. (6), which represents the typical form of model inversion control. If $\hat{\boldsymbol{h}}(\boldsymbol{x}, t)$ $=\boldsymbol{h}(\boldsymbol{x}, t), \dot{\boldsymbol{x}}$ in Eq. (3) is identical to $\boldsymbol{v}$. However, it is difficult to establish $\boldsymbol{h}(\boldsymbol{x}, t)$ exactly.

$$
\begin{aligned}
\boldsymbol{v} & \equiv \hat{\boldsymbol{h}}(\boldsymbol{x}, t)+\hat{\boldsymbol{B}} \boldsymbol{u} \\
\boldsymbol{u} & =\hat{\boldsymbol{B}}^{+}[\boldsymbol{v}-\hat{\boldsymbol{h}}(\boldsymbol{x}, t)]
\end{aligned}
$$

where,

$$
\hat{\boldsymbol{B}}^{+}=\left(\hat{\boldsymbol{B}}^{\mathrm{T}} \hat{\boldsymbol{B}}\right)^{-1} \hat{\boldsymbol{B}}^{\mathrm{T}}
$$

Here, the unknown $\hat{\boldsymbol{h}}(\boldsymbol{x}, t)$ can be defined based on the time delay estimation, as shown in Eq. (7). The sampling time step, $L$, is assumed to be very small and have little difference between the previous and current values for very small magnitudes.

$$
\hat{\boldsymbol{h}}(\boldsymbol{x}, t) \cong \boldsymbol{h}(\boldsymbol{x}, t-L)
$$

Applying Eq. (7), Eq. (3) can be rearranged as:

$$
\hat{\boldsymbol{h}}(\boldsymbol{x}, t) \cong \boldsymbol{h}(\boldsymbol{x}, t-L)=\dot{\boldsymbol{x}}(t-L)-\hat{\boldsymbol{B}} \boldsymbol{u}(t-L)
$$

Substituting Eq. (8) into Eq. (6) eliminates $\hat{\boldsymbol{h}}(\boldsymbol{x}, t)$ and yields the inversed control input as:

$$
\boldsymbol{u}=\boldsymbol{u}(t-L)+\hat{\boldsymbol{B}}^{+}[\boldsymbol{v}(t)-\dot{\boldsymbol{x}}(t-L)]
$$

Similar to determining the model inversion control, a feasible pseudo control requires error dynamics, such as Eq. (10). The objective of this control is to make the error converge to zero. Therefore, the desired error dynamics introduces a stable matrix, $\boldsymbol{A}_{d}$ :

$$
\dot{\boldsymbol{e}}_{d}=\dot{\boldsymbol{x}}_{d}-\dot{\boldsymbol{x}}=\boldsymbol{A}_{d} \boldsymbol{e}_{d}
$$

Assuming $\hat{\boldsymbol{h}}(\boldsymbol{x}, t)=\boldsymbol{h}(\boldsymbol{x}, t)$, and replacing $\dot{x}$ with $\boldsymbol{v}$ in Eq. (10), the pseudo control input becomes:

$$
\boldsymbol{v}=\dot{\boldsymbol{x}}_{d}-\boldsymbol{A}_{d} \boldsymbol{e}
$$

Substituting Eq. (11) into Eq. (9) yields Eq. (12), which is of the same form as shown in Hsia and Gao. ${ }^{10)}$

$$
\boldsymbol{u}=\boldsymbol{u}(t-L)+\hat{\boldsymbol{B}}^{+}\left[\dot{\boldsymbol{x}}_{d}-\boldsymbol{A}_{d} \boldsymbol{e}_{d}-\dot{\boldsymbol{x}}(t-L)\right]
$$

Furthermore, the desired dynamics can be defined as Eq. (13) applying the same stable $\boldsymbol{A}_{d}$ of the error dynamics

$$
\dot{\boldsymbol{x}}_{d}=\boldsymbol{A}_{d} \boldsymbol{x}_{d}+\boldsymbol{B}_{d} \boldsymbol{r}
$$

Substituting Eq. (13) into Eq. (12) yields Eq. (14), which is of the same form as shown in Youcef-Toumi and Ito ${ }^{8)}$.

$$
\boldsymbol{u}=\boldsymbol{u}(t-L)+\hat{\boldsymbol{B}}^{+}\left[\boldsymbol{A}_{d} \boldsymbol{x}+\boldsymbol{B}_{d} \boldsymbol{r}-\dot{\boldsymbol{x}}(t-L)\right]
$$

\subsection{TDC stability overview}

In this section, a brief overview of TDC stability is presented. Combining Eq. (3) and Eq. (9), the closed loop dynamics can be as Eq. (15)

$$
\dot{\boldsymbol{x}}=\boldsymbol{h}(\boldsymbol{x}, t)+\hat{\boldsymbol{B}} \boldsymbol{u}(t-L)+\hat{\boldsymbol{B}} \hat{\boldsymbol{B}}^{+}[\boldsymbol{v}(t)-\dot{\boldsymbol{x}}(t-L)]
$$

Applying Eq. (8) to Eq. (15) and if $\hat{\boldsymbol{B}} \hat{\boldsymbol{B}}^{+}=\boldsymbol{I}$, the closed loop dynamics can be defined as:

$$
\dot{\boldsymbol{x}}=\varepsilon(\boldsymbol{x}, t)+\boldsymbol{v}
$$

where

$$
\boldsymbol{\varepsilon}(\boldsymbol{x}, t)=\boldsymbol{h}(\boldsymbol{x}, t)-\boldsymbol{h}(\boldsymbol{x}, t-L) \quad \text { and } \quad \boldsymbol{v}=\boldsymbol{A}_{d} \boldsymbol{x}+\boldsymbol{B}_{d} \boldsymbol{r} .
$$

As presented, the TDC stability takes two main assumptions such that $L$ is sufficiently small and $\hat{\boldsymbol{B}}$ is a proper estimation of $\boldsymbol{B}(\boldsymbol{x}, t)$ including the condition, $\hat{\boldsymbol{B}} \hat{\boldsymbol{B}}^{+}=\boldsymbol{I}$.

First, the condition, $\hat{\boldsymbol{B}} \hat{\boldsymbol{B}}^{+}=\boldsymbol{I}$, Youcef-Toumi and Ito $^{8)}$ proved that the matching condition is always satisfied when the plant system is represented in the phase variable (canonical) form.

Second, Youcef-Toumi and $\mathrm{Wu}^{12)}$ proved that if $L \rightarrow 0$ and if $\left\|\boldsymbol{I}-\boldsymbol{B}(\boldsymbol{x}, t) \hat{\boldsymbol{B}}^{-1}\right\|<1$, then the closed loop dynamics is stable. In addition, in Eq. (16), it can be seen that, if $L \rightarrow 0$, then $\varepsilon \rightarrow 0$, the closed loop dynamics follows the stable desired dynamics $\boldsymbol{A}_{d}, \boldsymbol{B}_{d}$ and the error goes to zero 
in steady state. However, defining the range of sufficient small $L$ is highly complicated because it is related with $\boldsymbol{h}(\boldsymbol{x}, t)$, which is time variant uncertain nonlinear dynamics. For simplification, Youcef-Toumi and Ito $^{7)}$ suggested that the range of $L$ could be $L \leq 0.1 / \omega_{b}$ in a linear dynamics case. $\omega_{b}$ is the maximum possible bandwidth of the plant.

In general, the UAV flight control computer has fixed update rates due to limited computing power. Therefore, this paper focuses on the practical application of TDC to aircraft and examines the performance of applied TDC with given sample time $L(0.025 \mathrm{~s})$.

\section{Stability/Control Augmentation System (SCAS)}

In this section, the details of TDC-based pitch and roll SCAS design ${ }^{13)}$ are discussed. Since the equations for isolated pitch and roll are in the phase variable (canonical) form, they satisfy the matching condition. Furthermore, these equations are simple. Therefore, they are suitable for the implementation of TDC.

\subsection{Pitch SCAS}

Pure pitch dynamics in the longitudinal motion can be defined as:

$$
\dot{\theta}=q, \quad \dot{q}=\frac{1}{I_{y}} \bar{M}\left(q, \delta_{e}, \alpha, \dot{\alpha}, \ldots\right)
$$

where $\bar{M}$ is the total pitching moment that is defined as a combination of various states and nonlinear tables. Therefore, it shows strong nonlinear characteristics. Equation (17) can be rearranged as a single formulation shown in Eq. (18), with disturbance, $\delta(t)$ :

$$
I_{y} \ddot{\theta}=M_{q} q+M_{\delta_{e}} \delta_{e}+M_{\alpha} \alpha+M_{\dot{\alpha}} \dot{\alpha}+\delta(t)+\ldots
$$

If the terms including the angle of attack and other terms can be considered as disturbances, Eq. (18) can be expressed as Eq. (19):

$$
\left\{\begin{array}{l}
\dot{\theta} \\
\ddot{\theta}
\end{array}\right\}=\left[\begin{array}{cc}
0 & 1 \\
0 & M_{q}^{\prime}
\end{array}\right]\left\{\begin{array}{l}
\theta \\
\dot{\theta}
\end{array}\right\}+\left[\begin{array}{c}
0 \\
M_{\delta_{e}}^{\prime}
\end{array}\right] \delta_{e}+\left[\begin{array}{c}
0 \\
\delta(\alpha, \dot{\alpha}, t, \ldots)
\end{array}\right]
$$

where, $M_{()}^{\prime}=M_{()} / I_{y}$.

On the other hand, the desired dynamics are defined as:

$$
\left\{\begin{array}{l}
\dot{\theta}_{d} \\
\ddot{\theta}_{d}
\end{array}\right\}=\left[\begin{array}{cc}
0 & 1 \\
-\omega_{n}^{2} & -2 \zeta \omega_{n}
\end{array}\right]\left\{\begin{array}{l}
\theta_{d} \\
\dot{\theta}_{d}
\end{array}\right\}+\left[\begin{array}{c}
0 \\
\omega_{n}^{2}
\end{array}\right] \theta_{r}
$$

where, $\theta_{r}$ is the reference pitch angle input.

Also, Eq. (20) is equivalent to Eq. (21):

$$
\ddot{\theta}_{d}+2 \zeta \omega_{n} \dot{\theta}_{d}+\omega_{n}^{2} \theta_{d}=\omega_{n}^{2} \theta_{r}
$$

where, $\zeta$ is the damping ratio and $\omega_{n}$ is the natural frequency of the desired dynamics.

Substituting the reference input, $\boldsymbol{r}$, and the control input, $\boldsymbol{u}$, in Eq. (14) with their corresponding symbols yields:

$$
\delta_{e}=\delta_{e}(t-L)+\hat{\boldsymbol{B}}^{+}\left[\boldsymbol{A}_{d} \boldsymbol{x}+\boldsymbol{B}_{d} \theta_{r}-\dot{\boldsymbol{x}}(t-L)\right]
$$

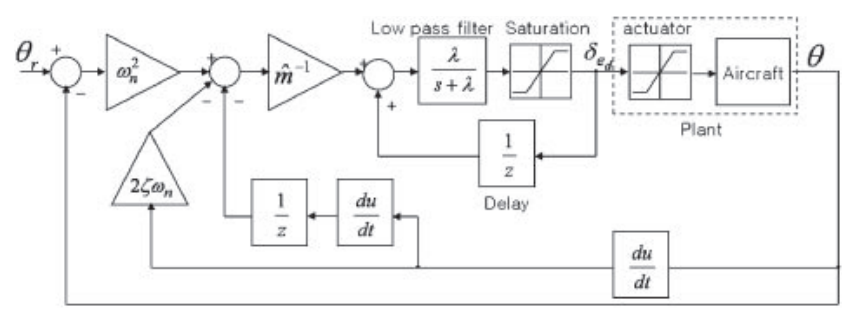

Fig. 1. Pitch channel SCAS.

where,

$$
\boldsymbol{A}_{d}=\left[\begin{array}{cc}
0 & 1 \\
-\omega_{n}^{2} & -2 \zeta \omega_{n}
\end{array}\right] \text { and } \boldsymbol{B}_{d}=\left[\begin{array}{c}
0 \\
\omega_{n}^{2}
\end{array}\right]
$$

Substituting $\boldsymbol{x}=\left[\begin{array}{ll}\theta & \dot{\theta}\end{array}\right]^{\mathrm{T}}$ and $\hat{\boldsymbol{B}}=\left[\begin{array}{ll}0 & \hat{M}_{\delta_{e}}^{\prime}\end{array}\right]^{\mathrm{T}}$ into Eq. (22) results in:

$$
\delta_{e}=\delta_{e}(t-L)+\hat{m}^{-1}\left[-\ddot{\theta}(t-L)+\omega_{n}^{2}\left(\theta_{r}-\theta\right)-2 \zeta \omega_{n} \dot{\theta}\right]
$$

where, $\hat{m}=\hat{M}_{\delta_{e}} / \hat{I}_{y} \quad$ and $\quad \hat{\boldsymbol{B}}^{+}=\left(\hat{\boldsymbol{B}}^{\mathrm{T}} \hat{\boldsymbol{B}}\right)^{-1} \hat{\boldsymbol{B}}^{\mathrm{T}}$.

In Eq. (23), the only property required from the plant model is the estimated control effectiveness, $\hat{m}$, which can be obtained by offline calculation. The other terms are all known.

Figure 1 shows the pitch SCAS of TDC. Both the damping ratio and the natural frequency of desired dynamics should be determined during tuning. The controller includes a low-pass filter and saturation blocks. The saturation block prevents the control actuator windup. ${ }^{15)}$ The low-pass filter is necessary to eliminate the high-frequency terms induced by the double derivative of $\theta .^{3}$ )

\subsection{Roll SCAS}

The roll channel of TDC SCAS can be designed in the same manner as described in the previous section. The pure roll channel dynamics can be defined as:

$$
\dot{\phi}=p, \quad \dot{p}=\frac{1}{I_{x}} \bar{l}\left(p, \delta_{a}, \beta, \ldots\right)
$$

The TDC control law for the roll channel can be obtained as:

$$
\delta_{a}=\delta_{a}(t-L)+\hat{l}^{-1}\left[-\ddot{\phi}(t-L)+\omega_{n}^{2}\left(\phi_{r}-\phi\right)-2 \zeta \omega_{n} \dot{\phi}\right]
$$

where, $\hat{l}=\hat{l}_{\delta_{a}} / \hat{I}_{x}$.

Table 1 shows the SCAS design parameters for pitch and roll.

\section{Auto-landing Guidance}

The design of the external loop for auto-landing guidance is discussed in this section. The longitudinal altitude control

Table 1. SCAS designed parameters.

\begin{tabular}{llcc}
\hline & \multicolumn{1}{c}{ Design parameter } & Pitch & Roll \\
\hline$\zeta$ & Desired damping ratio (ND) & 1.2 & 1.2 \\
$\omega_{n}$ & Desired natural frequency (rad/s) & 3 & 4 \\
$\lambda$ & Filter cut-off frequency (rad/s) & 10 & 10 \\
\hline
\end{tabular}


employs the TDC-based guidance law, ${ }^{13}$ ) while the lateral heading angle control is based on the LOS technique.

\subsection{Glide slope guidance}

The glide slope trajectory is a straight line with a given flight path angle. The altitude for the path can be defined as:

$$
\dot{h}=V \sin \gamma \cong V \gamma
$$

Employing the TDC concept, Eq. (26) can be reformulated as Eq. (27), and $\gamma$ is considered as an input.

$$
\dot{h}=\hat{f}+\hat{V} \gamma
$$

where, $\hat{f}=V \sin \gamma-\hat{V} \gamma+\delta(t)$.

If the desired error dynamic is defined as,

$$
\dot{e}_{d}=-\frac{1}{\tau} e_{d}
$$

which is exponentially stable, then:

$$
\dot{h}_{d}-\dot{h}=\dot{h}_{d}-\hat{V} \gamma-\hat{f}=-\frac{1}{\tau}\left(h_{d}-h\right)
$$

Appling time delay estimation (TDE) to Eq. (27), $\hat{f} \cong \hat{f}(t-L)=\dot{h}(t-L)-\hat{V} \gamma(t-L)$ and substituting into Eq. (28), then the TDC glide slope guidance control can be obtained as:

$$
\gamma_{\text {glide }}=\gamma_{\text {glide }}(t-L)+\hat{V}^{-1}\left[\dot{h}_{d}-\dot{h}(t-L)+\frac{1}{\tau}\left(h_{d}-h\right)\right]
$$

The block diagram of the glide slope guidance law is presented in Fig. 2.

\subsection{Flare guidance}

The desired flare altitude can be expressed as an exponential function ${ }^{16)}$ :

$$
h_{d_{\text {flare }}}=h_{0_{\text {flare }}} e^{-t / \tau}
$$

Applying $\dot{h}=V \sin \gamma \cong V \gamma$, Eq. (30) can be a solution to Eq. (31):

$$
\dot{h}=V \gamma=-h / \tau
$$

Finally, the desired flare flight path angle can be determined as:

$$
\gamma_{d_{\text {flare }}}=-\frac{h}{V \tau}
$$

The criterion for switching from the glide slope phase to the flare phase is determined in terms of the altitude.

\subsection{Lateral guidance}

The lateral guidance is implemented as a heading control. First, the heading controller is designed before dealing with the guidance to the target point. Figure 3 depicts a common heading controller using roll angle control. ${ }^{16)}$

In Figs. 3 and 5, the current heading $(\psi)$, which is fed back into this system, requires the 'course angle' $\left(\Psi^{\prime}\right)$ during the flight. On the other hand, this system requires the 'true heading' $(\Psi)$ during the landing run because the nose heading should be aligned with the runway. Therefore, a feedback heading-switch is required between the flight phase and the

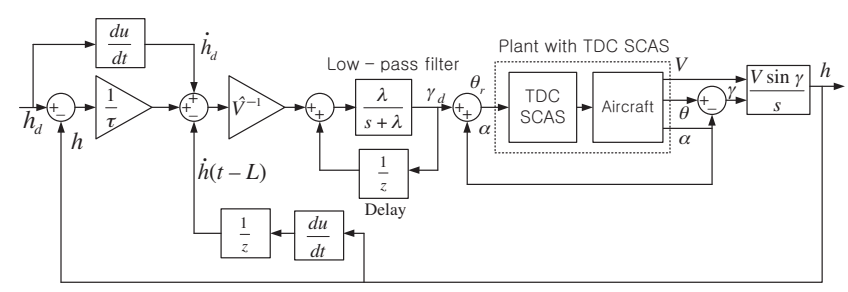

Fig. 2. Longitudinal glide slope guidance law.

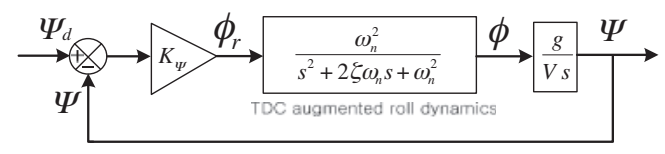

Fig. 3. Heading controller. ${ }^{16)}$

ground phase. The present paper assumes that the yaw angle equals the true heading.

During the ground run, the nose heading should be controlled using a nose gear steering on the runway. To perform this, the nose gear is mechanically linked with the rudder and the rudder moves proportionally to the roll command. Connecting rudder to roll command is a general control method to reduce adverse yaw due to roll during the flight.

The heading controller shown in Fig. 3 assumes that the 2nd-order system inside is the desired roll dynamics of Eq. (19), which is augmented dynamics by the implementation of the TDC SCAS. Using this simplification, the gain, $K_{\psi}$ for the heading control can be easily determined.

The root locus generated by the system in Fig. 3 is shown in Fig. 4. This indicates that the system is stable for $K_{\psi}$ less than 1.3. The present paper selects 0.7 for the gain value.

With the stable heading control loop being established, the next task is to design heading generation for guidance to the target point. The heading generation employs the LOS technique shown in Fig. 5. The figure depicts the geometry of the lateral guidance that accounts for crosswind.

To simplify the problem, the figure shows the situation when the azimuth of the reference line $\psi_{\text {ref }}$ equals 0 . In the figure, the line of sight is designated by $\Lambda$, the deviation distance from the reference line by $d$, and the distance to the target by $R$. The symbols within parentheses denote the definition of the direction.

To design a control that makes $d$ exponentially smaller, the desired dynamics of $d$ can be defined as Eq. (33), similar to Eq. (30):

$$
d_{d}=d_{0} e^{-t / \tau}
$$

By applying $\dot{d}=V \sin \psi_{\text {rel }} \cong V \psi_{\text {rel }}$, Eq. (33) is solved as:

$$
\dot{d}=V \psi_{\text {rel }}=-d / \tau
$$

Finally, the desired heading angle is determined as:

$$
\psi_{\mathrm{rel}_{d}}=-\frac{d}{V \tau}
$$

It is notable that Eq. (35) does not have range $R$ in the denominator compare with the geometric LOS guidance law, $\psi_{\text {rel }_{d}}=-K_{\mathrm{LOS}} \tan ^{-1}(d / R)$, then Eq. (35) refines the prob- 


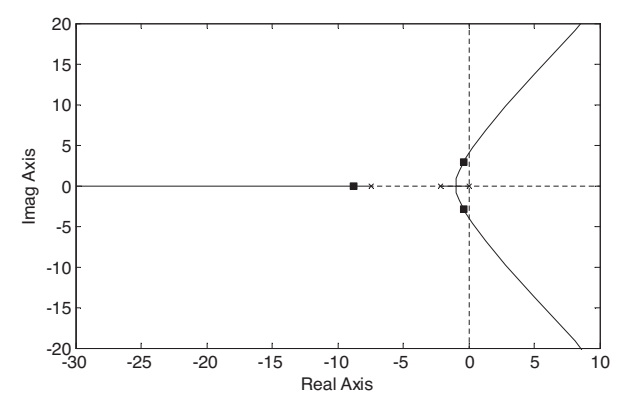

Fig. 4. Heading controller root locus $\left(K_{\psi}=0.7\right)$.

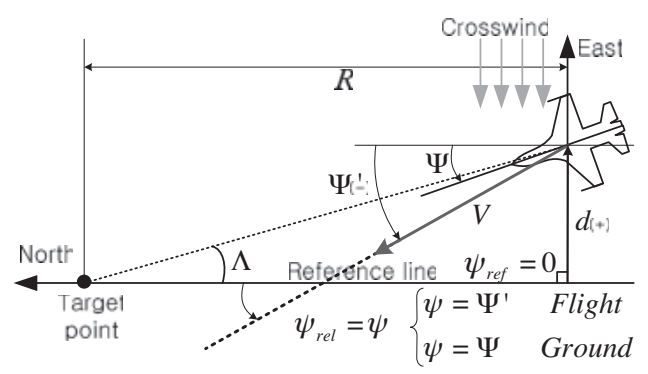

Fig. 5. Lateral guidance geometry.

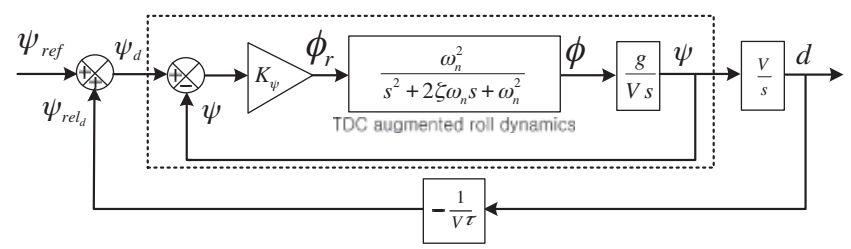

Fig. 6. Lateral guidance law.

lem that the loop gain goes to infinity as the range goes to zero while the aircraft approaches near to the target. ${ }^{16)}$

Finally, as shown in Fig. 6, the actual heading guidance command is determined as a sum of the desired heading and the azimuth of the reference line:

$$
\psi_{d}=\psi_{\mathrm{rel}_{d}}+\psi_{\mathrm{ref}}
$$

\section{Modeling and Simulation}

Figure 7 shows Electric Aerial Vehicle-1 Auto Landing (EAV-1 AL), the test bed of the controller.

An aircraft simulation model is developed in the MATLAB/Simulink environment. The refresh rate of the simulation and controller is set to $40 \mathrm{~Hz}$. Therefore, the time step, $L$, in Eq. (7) becomes 0.025 . The aerodynamic data is calculated using DATCOM, and the landing gear model for auto-landing has a tire model with a simple 1st-order spring and damper setup. ${ }^{17)}$ The landing gear model accounts for friction in the longitudinal and lateral directions of the gear wheel so that steering can be simulated. The implemented dynamics model is shown in Fig. 8.

\subsection{Crosswind landing simulation}

Figure 9 shows the 3D trajectory of the TDC-based autolanding guidance simulation. A steady $3 \mathrm{~m} / \mathrm{s}$ crosswind is applied, starting $2 \mathrm{~s}$ after the beginning of the simulation.

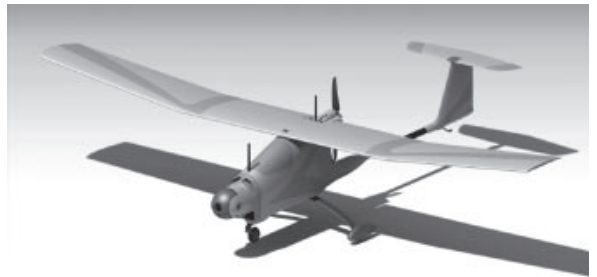

Fig. 7. EAV-1 AL.

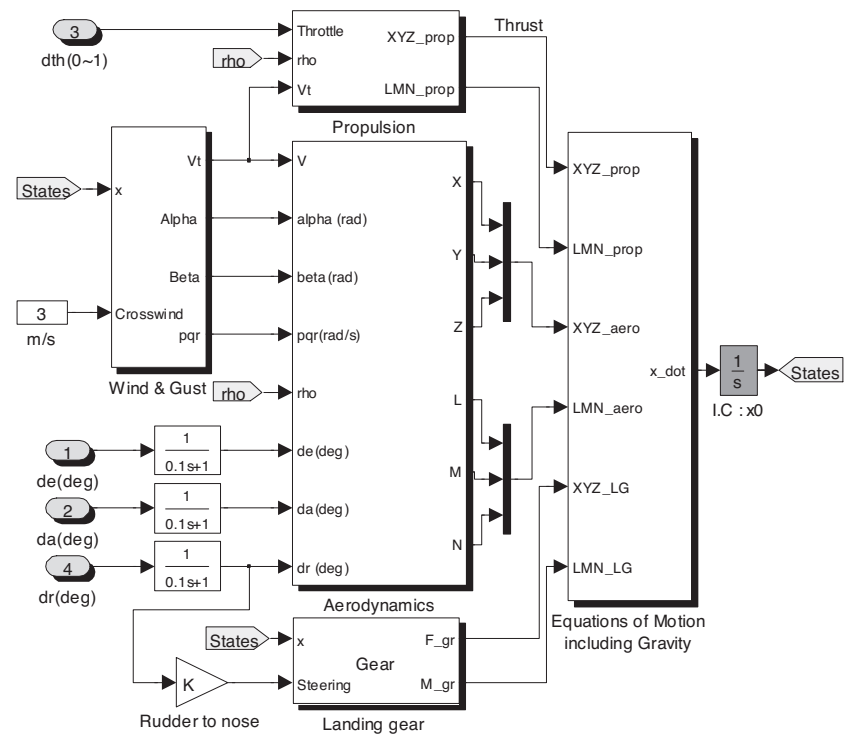

Fig. 8. Nonlinear simulation block diagram.

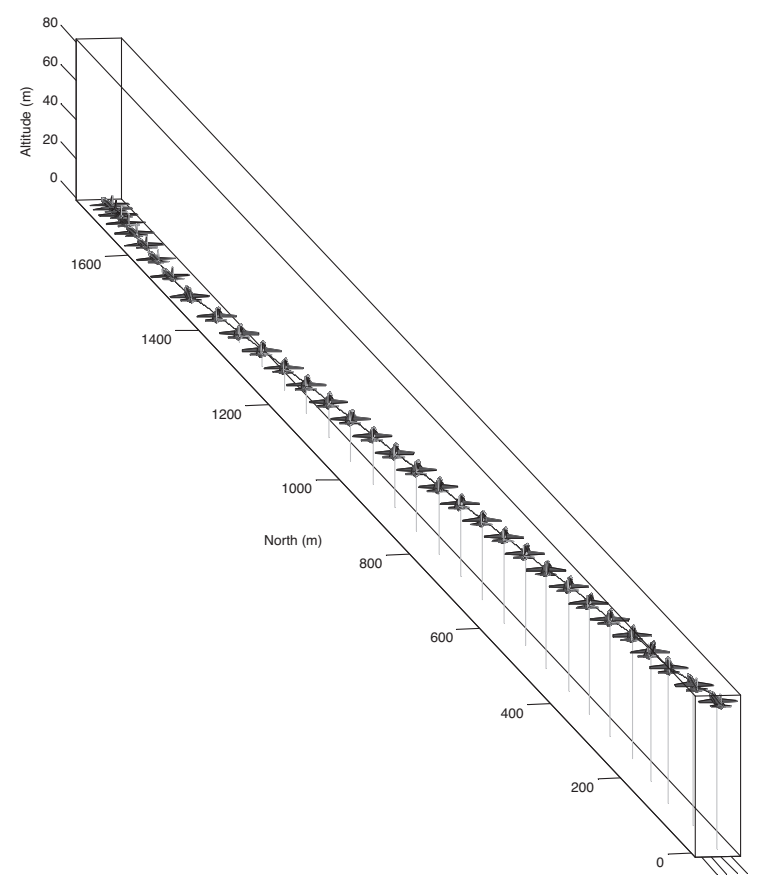

Fig. 9. Crosswind landing flight path (crab, de-crab maneuver).

The feedback heading-switch results in the observable crab and de-crab maneuvers under the crosswind. The flare altitude is set to $1.36 \mathrm{~m}$, which is half the wingspan. The idle setting and the heading-switch for touchdown occur at the flare altitude. 
Trans. Japan Soc. Aero. Space Sci., Vol. 58, No. 1, 2015
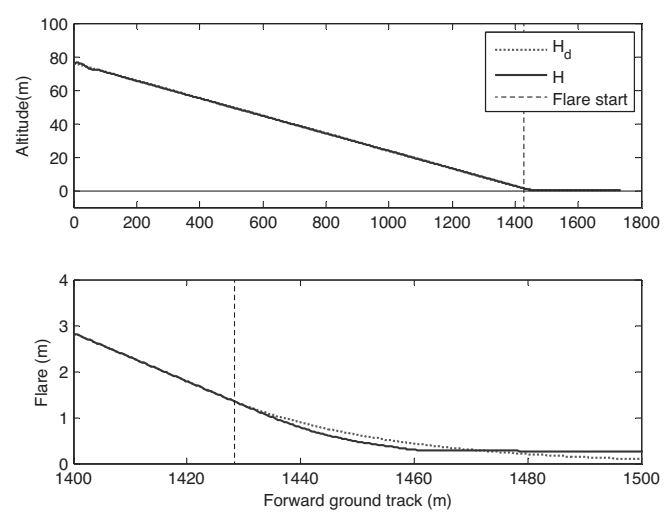

Fig. 10. Longitudinal landing flight path.
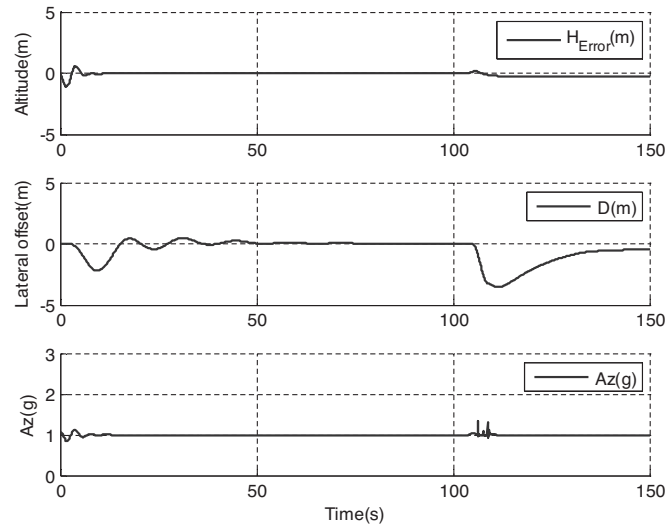

Fig. 12. Tracking error and touchdown impact.

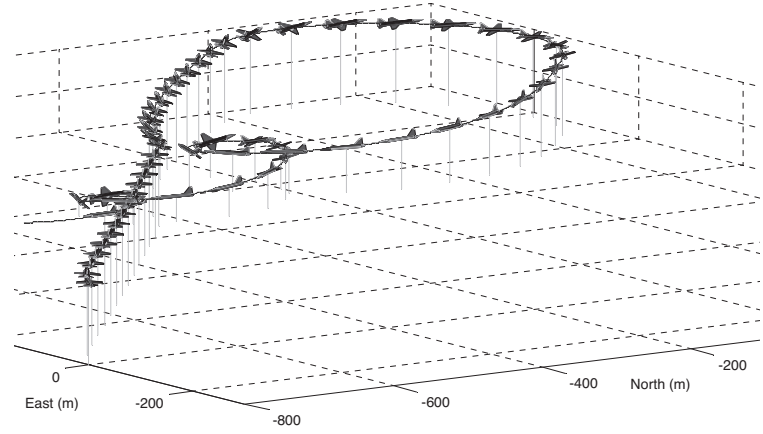

Fig. 14. PID SCAS auto-landing trajectory.
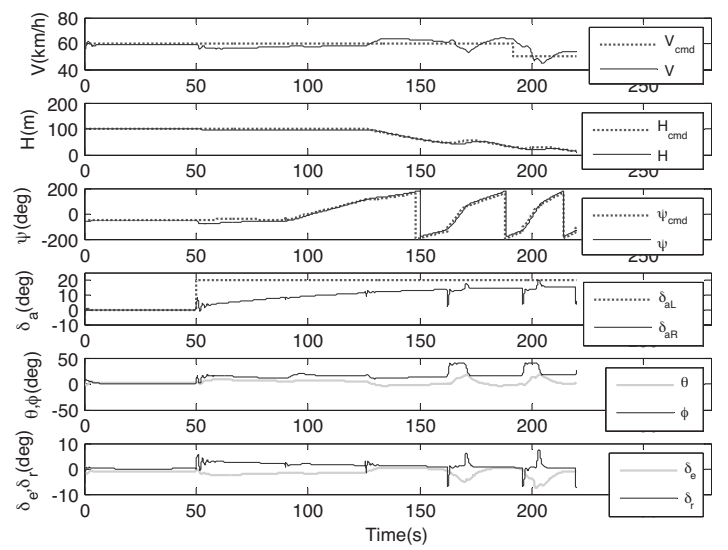

Fig. 16. PID SCAS auto-landing time history.
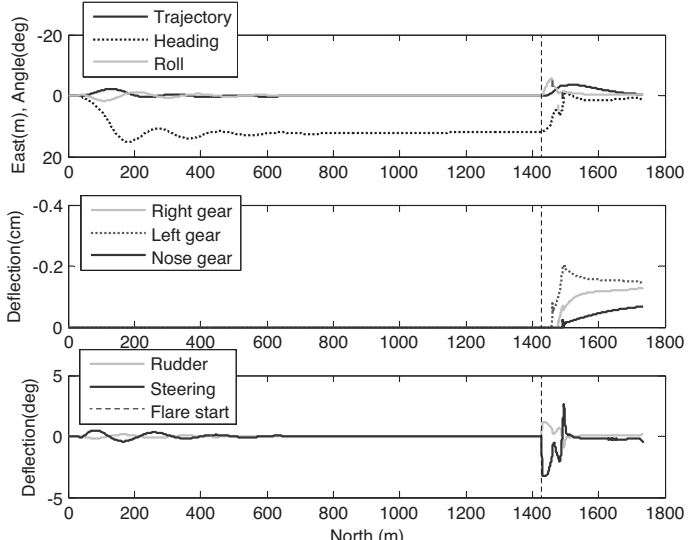

Fig. 11. Lateral landing flight path and associated parameters
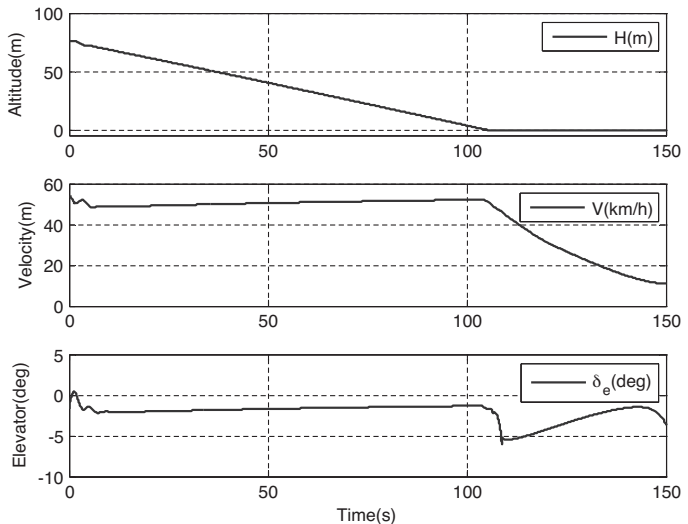

Fig. 13. Primary longitudinal parameters.

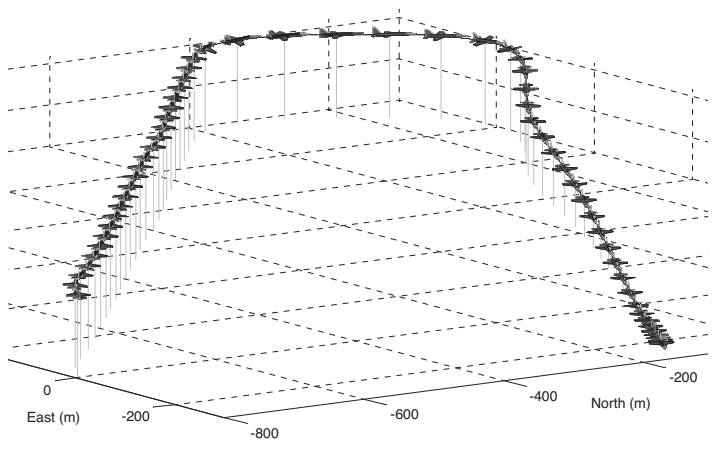

Fig. 15. TDC SCAS auto-landing trajectory.
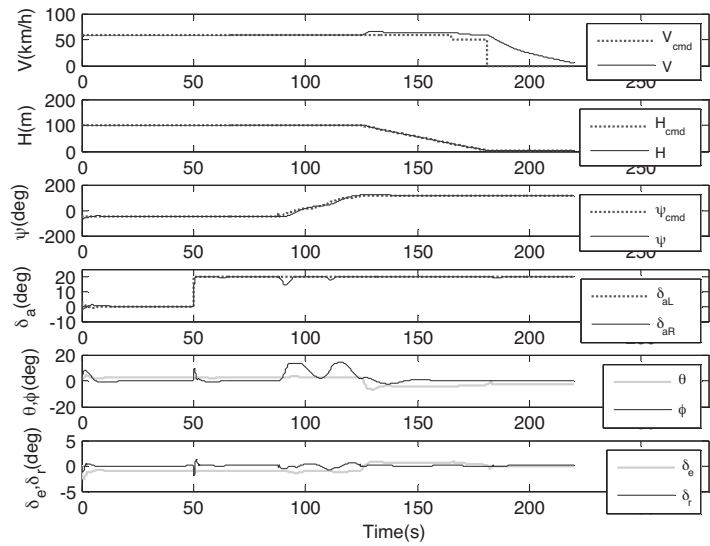

Fig. 17. TDC SCAS auto-landing time history. 
Figure 10 shows the time histories of the longitudinal auto-landing and flare, and Fig. 11 shows the time histories of the lateral attitudes, landing gear deflection and control angles. Figure 12 is the time histories of the longitudinal and lateral trajectory tracking errors and the impact acceleration during landing. Figure 13 shows the time histories of the altitude, speed and the longitudinal control angle.

\subsection{Landing simulation with aileron fault}

The fault of an aileron is simulated here. For a case where the left aileron is stuck at a $20^{\circ}$ angle downward at simulation time $50 \mathrm{~s}$, PID control and TDC control are compared in the auto-landing performance. The simulation consists of a turn, followed by the landing of the aileron-faulted aircraft.

Figure 14 shows the landing trajectory of the aircraft with PID-based auto-landing guidance. The PID SCAS is designed using CONDUIT. ${ }^{18)}$ As the fault of the aileron results in rolling moment to the right, the aircraft cannot make a left turn for landing after the first right turn. As a consequence, a safe landing is not achievable.

Figure 15 shows the landing trajectory of the aircraft with TDC-based auto-landing guidance. It is remarkable that, for the same fault condition, the aircraft makes a reasonable landing.

Figures 16 and 17 show the time histories associated with the PID and TDC controllers, respectively.

\section{Conclusion}

Employing the TDC technique, the present study proposed a design procedure for the auto-landing guidance of an unmanned aerial vehicle (UAV) in both longitudinal and lateral directions. TDC-based controllers for pitch, roll and altitude were designed. The lateral guidance adopted the LOS guidance method with the inside loop of TDC-controlled roll dynamics.

The TDC-based auto-landing guidance was validated with the nonlinear simulations for overall landing elements including glide slope, flare and touchdown using a simulation model which included the landing gear model. It was shown that, for the design of control loops which account for the influence of crosswind, crab and de-crab maneuvers can be successfully carried out during the landing procedure by switching the heading feedback. In addition, a comparison simulation for a case with a faulty aileron showed that TDC SCAS is robust and fault tolerant.

Additionally, through all the simulations, the applied TDC controller shows satisfactory performance in the case that the time step $L$ is $0.025 \mathrm{~s}$. It confirms that the smaller time step also guaranties satisfactory performance.

\section{References}

1) Lee, C.-H., Kim, T.-H., and Tahk, M.-J.: Missile Autopilot Design for Agile Turn Using Time Delay Control with Nonlinear Observer, Int. J. Aeronaut. Space Sci., 12 (2011), pp. 266-273.

2) Park, B.-G., Kim, T.-H., and Tahk, M.-J.: Time-Delay Control for Integrated Missile Guidance and Control, Int. J. Aeronaut. Space Sci., 12 (2011), pp. 260-265.

3) Chang, P. H., Park, B. S., and Park, K. C.: An Experimental Study on Improving Hybrid Position Force Control of a Robot Using Time Delay Control, Mechatronics, 6 (1996), pp. 915-931.

4) Chin, S. M., Lee, C. O., and Chang, P. H.: An Experimental Study on the Position Control of an Electrohydraulic Servo System Using Time Delay Control, Control Eng. Practice, 2 (1994), pp. 41-48.

5) Chang, P. H., Kim, D. S., and Park, K. C.: Robust Force/Position Control of a Robot Manipulator Using Time-Delay Control, Control Eng. Practice, 3 (1995), pp. 1255-1264.

6) Morgan, R. G. and Ozguner, U.: A Decentralized Variable Structure Control Algorithm for Robotic Manipulators, IEEE J. Robotics Autom., 1 (1985), pp. 57-65.

7) Youcef-Toumi, K. and Ito, O.: Controller Design for Systems with Unknown Dynamics, American Control Conference, 1987, pp. 836-845.

8) Youcef-Toumi, K. and Ito, O.: A Time Delay Controller Design for Systems with Unknown Dynamics, American Control Conference, 1988, pp. 904-913.

9) Hsia, T. C.: A New Technique for Robust Control of Servo Systems, IEEE Trans. Ind. Electronics, 36 (1989), pp. 1-7.

10) Hsia, T. C. and Gao, L. S.: Robot Manipulator Control Using Decentralized Linear Time-Invariant Time-Delayed Joint Controllers, IEEE International Conference on Robotics and Automation, 1990, pp. 2070-2075.

11) Chang, P. H. and Lee, J. W.: A Model Reference Observer for TimeDelay Control and Its Application to Robot Trajectory Control, IEEE Trans. Control Syst. Technol., 4 (1996), pp. 2-10.

12) Youcef-Toumi, K. and Wu, S.-T.: Input/Output Linearization Using Time Delay Control, ASME J. Dynam. Syst. Meas. Control, 114 (1992), pp. 10-19.

13) Choi, H. S., Lee, S., Lee, J., Kim, E. T., and Shim, H.: Aircraft Longitudinal Auto-Landing Guidance Law Using Time Delay Control Scheme, Trans. Jpn. Soc. Aeronaut. Space Sci., 53 (2010), pp. 207-214.

14) Choi, H. S.: Research on the UAV Model Based Design and Adaptive Control for Uncertain Dynamics, Ph.D. Thesis, KAIST, 2011, pp. 95-97 (in Korean).

15) Chang, P. H. and Park, S. H.: On Improving Time-Delay Control under Certain Hard Nonlinearities, Mechatronics, 13 (2003), pp. 393-412.

16) Blakelock, J. H.: Automatic Control of Aircraft and Missiles, 2nd ed., John Willey \& Sons, New York, 1991, pp. 88-97, 178-179.

17) Atkinson, C. J.: Development of an Aerodynamic Table Lookup System and Landing Gear Model for the Cal Poly Flight Simulator, http:// aerosim.calpoly.edu/library/senoir-projects, 2002.

18) Lee, S., Lee, J., and Lee, D. S.: Lateral and Directional SCAS Controller Design Using Multidisciplinary Optimization Program, J. Korean Soc. Aeronaut. Space Sci., 40 (2012), pp. 251-257 (in Korean). 